\title{
Effect of Short Chain Aliphatic Carboxylic Acids on Human Immunoglobulin $G$ in Non-buffered Medium
}

\author{
ULLA • B R I T T HA N S O N
}

Department of Clinical Chemistry, University of Lund, Malmö General Hospital, S-214 01 Malmö, Sweden

\begin{abstract}
The effect, at acid pH, of unbranched short chain carboxylic acids on human immunoglobulin G (IgG), was investigated with ultracentrifugal analysis, and antigen-antibody crossed electrophoresis. Native IgG formed small amounts of aggregates when dissolved in $0.1 \mathrm{M}$ solutions of carboxylic acids and larger amounts of aggregates when back dialysed to neutrality. Acid samples were electrophoretically more heterogeneous than neutral samples. No correlation was found between the amount of aggregates formed and the carbon chain length of carboxylic acid added. The largest amount of aggregates was formed when IgG was frozen after acidification with carboxylic acids. The amount of aggregates then increased with increasing carbon-chain length of carboxylic acid.

Aggregates formed by freezing of untreated IgG in neutral medium dissociated, wholly or partially, in $0.05-0.25 \mathrm{M}$ formic acid. Higher concentrations $(0.50-1.25 \mathrm{M})$ of formic acid caused aggregation. The acid samples had a higher positive net charge than had the original sample dissolved in neutral buffer. On back dialysis of acid samples to neutrality aggregation occurred in all samples. The amount of aggregates increased with increasing molarity of the carboxylic acid. In $1 \mathrm{M}$ formic acid solution only $30 \%$ of the molecules existed in monomer form. Molecules with cathodal electrophoretic mobility seemed to be mainly responsible for the aggregation.
\end{abstract}

Tmmunoglobulin complexes formed in vivo and antigen-antibody aggregates dissociate in acid solution containing glycine. ${ }^{1-3}$ Also aggregates formed on freezing of immunoglobulin $\mathrm{G}(\mathrm{IgG})$ dissociate in this medium.4

IgG forms complexes when frozen in $0.1 \mathrm{M}$ acetic acid, but not when frozen in $0.1 \mathrm{M}$ glycine- $\mathrm{HCl} \mathrm{pH} \mathrm{3.0.4}$ Reduced and alkylated heavy chains of IgG are often separated from light chains in non-buffered solutions of short chain aliphatic carboxylic acids. Heavy chains form complexes when the $\mathrm{pH}$ of the carboxylic acid solution is increased to $7 .^{5}$ But no aggregation occurs if the carboxylic acid is replaced by glycine- $\mathrm{HCl} \mathrm{pH} 3.0$ before the $\mathrm{pH}$ is elevated. ${ }^{6}$ Heavy chains from rabbit $\operatorname{IgG}$, isolated from anti-adeno virus type 5 serum, more effectively inactivate the virus when dissolved in glycine- $\mathrm{HCl} \mathrm{pH} \mathrm{3.0,6}$

Acta Chem. Scand. 24 (1970) No. 6 
than when dissolved in propionic acid. ${ }^{7}$ Heavy chains obtained from rabbits not immunized with adeno virus also inactivate adeno virus when dissolved in glycine-HCl, in spite of the fact that IgG by itself has no inactivating effect. ${ }^{8}$

At neutral $\mathrm{pH}$ the aggregation of IgG on freezing is effectively inhibited by straight chain aliphatic amino acids. ${ }^{4}$ The inhibition decreases with increasing carbon chain length of added amino acid. ${ }^{4}$ At neutral $\mathrm{pH}$ sodium or ammonium salts of straight chain aliphatic carboxylic acids are almost as good inhibitors as straight chain aliphatic amino acids. ${ }^{9}$

In this paper the effect, at acid $\mathrm{pH}$, of unbranched short chain carboxylic acids on native and aggregated normal human IgG was studied with ultracentrifugal analysis and antigen-antibody crossed electrophoresis.

\section{MATERIALS AND METHODS}

Sera. Frozen sera from apparently healthy subjects were used.

Antiserum. Serum from rabbits immunized with purified IgG, prepared from a pool of normal human sera, dissolved in glycine-HCl, $\mathrm{pH} 3.0$, was used.

Antigen-antibody crossed electrophoresis (Ag-ab crossed electrophoresis) was performed according the method of Laurell. ${ }^{10}$

Isolation of the anodal part of IgA was made by agarose gel electrophoresis on cooled glass plates (3 mm layer, $1 \%$ agarose, $0.075 \mathrm{M}$ barbital buffer $\mathrm{pH} 8.4,2.0 \mathrm{mM}$ in respect of Ca-lactate, $20 \mathrm{~V} / \mathrm{cm}$ for $2 \mathrm{~h}$ ). IgG mixed in $1 \%$ agarose at $50^{\circ} \mathrm{C}$ was poured into a through $(5 \mathrm{~mm})$ in the agarose gel. The gel containing anodal IgG was cut out, homogenized at room temperature, and the protein was eluted with $0.2 \mathrm{M} \mathrm{NaCl}$ at the same temperature.

Dialysis against various solutions was continued for $48 \mathrm{~h}$. All reagents used were of analytical grade. Unless otherwise stated a phosphate- $\mathrm{NaCl}$ buffer ( $0.05 \mathrm{M}$ sodium phosphate $\mathrm{pH} 7.0,0.5 \mathrm{M}$ in respect of $\mathrm{NaCl}$ ) was used as neutral buffer.

Isolation of IgG, determination of protein concentration and of ratio between $7 S$ molecules and soluble aggregates was done as earlier described.11,12

The percental amount of precipitates $(p)$ was estimated from photographs obtained when the samples were run in an analytical ultracentrifuge with schlieren optics. All photographs were taken at the same angles. $p$ was calculated according to the following formula: $p\left(y_{1}-y_{2}\right) \times 100 / y_{1}$ where $y_{1}$ denotes the area representing soluble material in non-precipitated sample and $y_{2}$ the area representing soluble material in precipitated sample. Both samples were dissolved in solvent $1_{1}$, measured at time $t_{1}$.

\section{RESULTS}

The area of the sedimenting boundary, measured at the time $t_{1}$, of a protein in the concentration $c_{1}$, did not vary with the medium. The width of the boundary, defined as the ratio of the area, to the maximum height was, however, always lower when IgG was dissolved in acid medium than when it was dissolved in neutral medium.

Effect of carboxylicacids of different carbon chain lengths on native IgG

Results of ultracentrifugal analysis of samples in acid medium and after the samples had been dialysed against neutral buffer are given in Table 1. Unlike 0.1 M formic, acetic, and valeric acid, $0.1 \mathrm{M}$ propionic acid aggregated 
Table 1. Effect of carboxylic acids with different carbon chain lengths on native IgG 1, estimated by Analytical Ultracentrifuge.

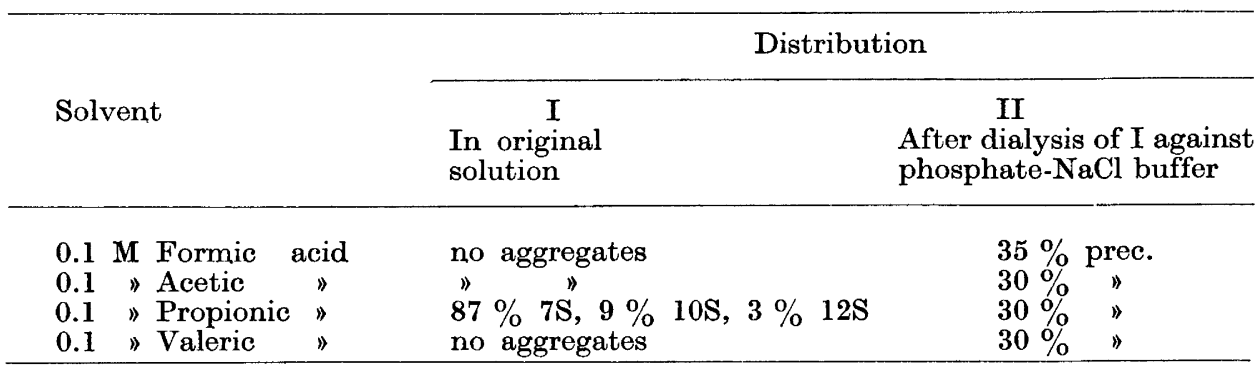

the native globulin (IgG 1). All samples contained precipitates in an amount $30 \%$ or more after dialysis against phosphate-NaCl buffer. There was no correlation between precipitation and carbon chain length of added carboxylic acid.

The results of ultracentrifugal analysis of another native $\operatorname{IgG}$ (IgG 2) treated with carboxylic acids with carbon-chains of different lengths are given in Table 2 . In this protein only traces of aggregates could be demonstrated in propionic acid. Also the sample dissolved in acetic acid now contained small amounts of aggregates.

Results of antigen-antibody crossed electrophoresis (Fig. 1). IgG 1 and 2 gave identical results. IgG 1 was chosen to represent both. The acidified samples showed a migration towards the cathode faster than untreated sample in neutral buffer (Fig. $1 \mathrm{~A}$ ). After the acid treated samples had been dialysed against neutral buffer (Fig. 1 B) they did not differ in charge distribution from untreated sample.

Fig. 1. Antigen-antibody crossed electrophoresis of native IgG 1 treated with $0.1 \mathrm{M}$ carboxylic acids of different carbon chain lengths, A; in acid medium, B; after back dialysis to neutrality.

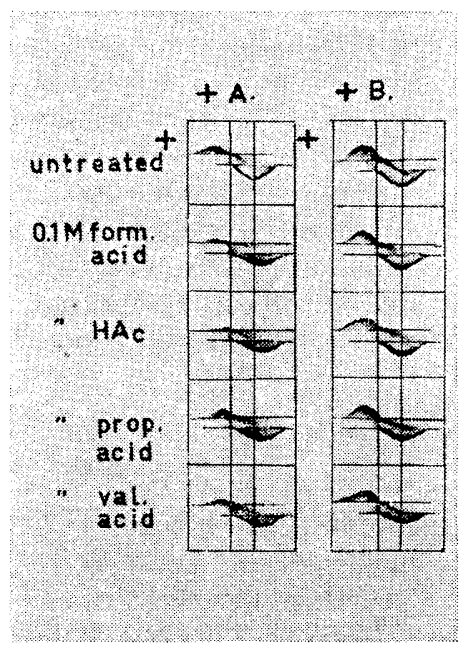

Acta Chem. Scand. 24 (1970) No. 6 
Comparison of the electrophoretic migration of an IgG with that of its isolated anodal part dissolved in $0.1 \mathrm{M}$ propionic acid revealed that following acidification some of the anodal molecules migrated towards the cathode. They migrated in cathodal direction just as far as, but not farther than, the molecules in the untreated IgG.

Effect of carboxylicacidsof different carbon chain lengths on IgG when frozen in acid medium

Results of ultracentrifugal analysis (Table 2). Those samples (IgG 2) that had been treated with $0.1 \mathrm{M}$ acids and analysed in the centrifuge were frozen (stored for $2 \mathrm{~h}$ at $-20^{\circ} \mathrm{C}$ and afterwards allowed to thaw at room tempera-

Table 2. Effect of carboxylic acids with different carbon chain lengths on IgG 2 when frozen in this medium, estimated by Analytical Ultracentrifuge.

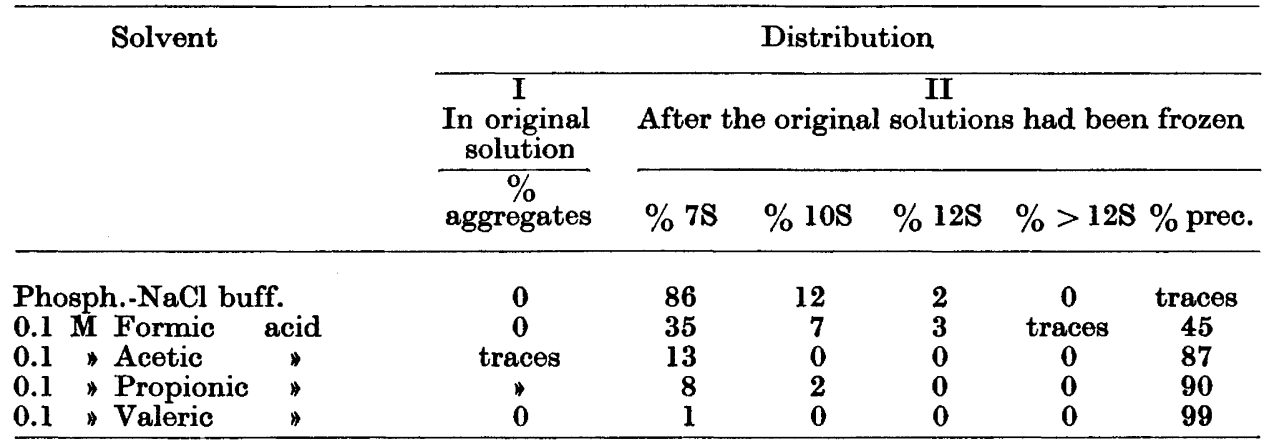

ture) ten times and analysed again. $14 \%$ of the molecules in the phosphate$\mathrm{NaCl}$ sample had then formed aggregates. The corresponding figure for the frozen samples containing acid was $45 \%$ or more. Aggregate formation increased with increasing carbon chain length of added carboxylic acid. In the valeric acid solution only $1 \%$ of the protein persisted in soluble form.

Results of antigen-antibody crossed electrophoresis of IgG 2, frozen in formic acid or neutral medium and unfrozen in neutral medium (Fig. 2). Unfrozen IgG at neutral $\mathrm{pH}$ migrated fastest towards the cathode and the sample frozen in acid medium migrated somewhat faster than that frozen in neutral medium.

Effect of formic acid of increasing concentration o n a g g r e gated IgG

Results of ultracentrifugal analysis (Fig. 3). An IgG (IgG 3) containing $10 \%$ of the molecules in the form of aggregates was used in the study. Only 7S molecules were demonstrated in the sample containing 0.05 and $0.10 \mathrm{M}$ formic acid. Also the $0.25 \mathrm{M}$ formic acid solution contained smaller percental amounts of aggregates than did the globulin before it was acidified. The 


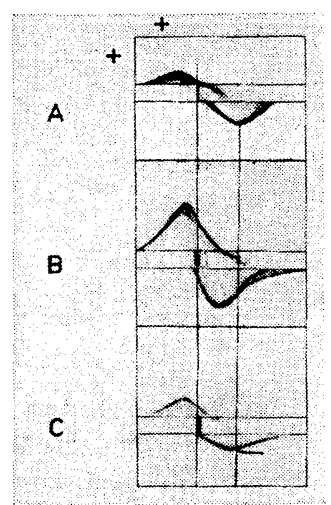

Fig. 2. Antigen-antibody crossed electrophoresis of IgG 2 frozen in $0.1 \mathrm{M}$ formic acid compared with IgG 2 frozen and unfrozen at $\mathrm{pH}$ 7.0, $\mathrm{A}$; unfrozen IgG at $\mathrm{pH}$ 7.0, B; IgG frozen at $\mathrm{pH} 7.0$, C; frozen in formic acid medium.

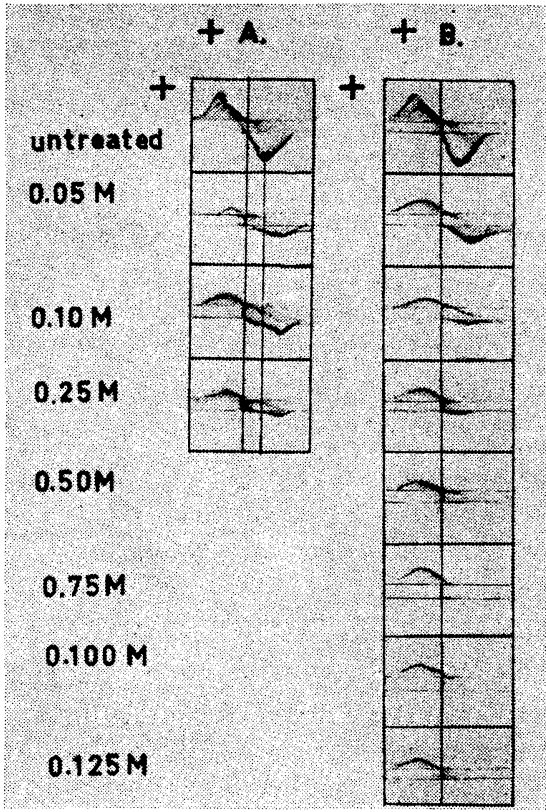

Fig. 3. Antigen-antibody crossed electrophoresis of IgG 3 denatured by freezing in neutral medium, dissolved in formic acid of increasing concentration, $A$; in acid medium, B; after back dialysis to neutrality.

percental amount of aggregates increased from 17 to 27 when the molarity of the acid solution was increased from 0.75 to 1.25 . Only soluble aggregates could be seen in the solutions. After the formic acid samples and the phosphate$\mathrm{NaCl}$ solution had been centrifuged once and dialysed against phosphate$\mathrm{NaCl}$, they were analysed again (Fig. 3, column 3). The phosphate-NaCl sample then contained $9 \%$ of the molecules as a $10 \mathrm{~S}$ component and $6 \%$ as a precipitate. The sample acidified with 0.05 and $0.1 \mathrm{M}$ formic acid contained approximately the same amount of aggregates as the globulins not dissolved in acid. The percental amount of aggregates increased from 40 to 69 when the molarity of acid was increased from 0.25 to 1.25 . In all solutions most of the aggregates existed as precipitates.

Result of antigen-antibody crossed electrophoresis of the acid samples accounted for in Table 3, column 2 (Fig. $3 \mathrm{~A}$ ). The samples acidified with $0.05-$ $0.25 \mathrm{M}$ formic acid migrated towards the cathode faster than the untreated sample. A double peak was seen in the anodal region in neutral as well as in acidified samples. The height of the anodal and that of cathodal peak decreased with increasing molarity of acid. After the samples had been dialysed against neutral buffer, they were analysed again (Fig. 3 B). The higher the molarity 
Table 3. Effect of increasing concentration of formic acid on IgG 3 aggregated by freezing at neutral $\mathrm{pH}$, estimated by Analytical Ultracentrifuge.

\begin{tabular}{|c|c|c|c|c|c|c|c|c|c|c|c|c|}
\hline \multirow{3}{*}{\multicolumn{3}{|c|}{ Solvent }} & \multicolumn{10}{|c|}{ Distribution } \\
\hline & & & \multicolumn{6}{|c|}{$\begin{array}{c}\text { I } \\
\text { In original solution }\end{array}$} & \multicolumn{4}{|c|}{$\begin{array}{l}\text { II } \\
\text { After dialysis of I against } \\
\text { phosphate-NaCl buffer. }\end{array}$} \\
\hline & & & $\% 7 \mathrm{~S}$ & $\% 10$ & $\% 12$ & $2 \mathrm{~S} \%>$ & $>12 \mathrm{~S}$ & $\%$ prec. & $\% 7 \mathrm{~S}$ & $\% 10 S$ & $\% 12 \mathrm{~S}$ & $\%$ prec. \\
\hline \multirow{2}{*}{\multicolumn{3}{|c|}{$\begin{array}{l}\text { Phosph.-NaCl buff. } \\
0.05 \mathrm{M} \text { Formic acid }\end{array}$}} & 90 & 10 & 0 & ) & 0 & 0 & 85 & 9 & 0 & 6 \\
\hline & & & 100 & 0 & 0 & 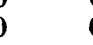 & 0 & 0 & 81 & 4 & 0 & 15 \\
\hline $0.10 \mathrm{M}$ & $"$ & $"$ & 100 & 0 & 0 & ) & 0 & 0 & 84 & 4 & 0 & 11 \\
\hline $0.25 \mathrm{M}$ & & $"$ & 95 & 5 & 0 & 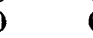 & 0 & 0 & 60 & 7 & 0 & 23 \\
\hline $0.50 \mathrm{M}$ & $"$ & $"$ & 83 & 14 & 3 & 3 & 0 & 0 & 45 & 3 & 0 & 52 \\
\hline $0.75 \mathrm{M}$ & " & $"$ & 70 & 19 & 9 & & 3 & 0 & 29 & 4 & 0 & 67 \\
\hline $1.00 \mathrm{M}$ & . & $\Rightarrow$ & 65 & 21 & 12 & & 3 & 0 & 32 & 4 & 0 & 64 \\
\hline $1.25 \mathrm{M}$ & . & . & 63 & 30 & 8 & 3 & 0 & 0 & 31 & 3 & 0 & 66 \\
\hline
\end{tabular}

of formic acid used for acidification, the lower was the height of the peak in the cathodal region. The height of the anodal peaks decreased at most slightly when the molarity of acid was increased. A double peak was seen in the anodal region also after neutralization.

\section{DISCUSSION}

The areas below the 7S peaks, seen on ultracentrifugal analysis of the samples, did not vary with the medium used. The widths of the bounderies were, however, always smaller when IgG was dissolved in acid medium compared with when dissolved in neutral medium. On ultracentrifugal analysis the protein thus seemed to be less polydisperse in carboxylic acid solution than in neutral medium. Ross and Jirgensons ${ }^{13}$ studied the rotatory dispersion of two IgG's in acid medium and produced evidence of formation of right handed $\alpha$-helices. Studies of the structures of human IgG, reviewed by Stanworth and Pardoe, ${ }^{14}$ show that this protein probably lacks helical conformation at neutral $\mathrm{pH}$. Parts of IgG might, like poly-L-glutamic acid studied by Urnes and Doty, ${ }^{15}$ assume a helical configuration in carboxylic acid solution and are converted to a random coil at neutral $\mathrm{pH}$.

On electrophoresis acidified material (except when frozen) migrated a longer distance in cathodal direction than non-acidified IgG but an equally long distance in anodal direction as untreated IgG. As parts of the same sample were studied in both acid and neutral medium, and as the amount of precipitates could be ignored, the same molecules were studied in both media. Thus, IgG treated with carboxylic acid was electrophoretically more heterogeneous than untreated IgG. The increase in positive net charge seen at acid $\mathrm{pH}$ might be explained by carboxylate side chains in the protein being partly neutralized at $\mathrm{pH}$ 3.0. 
Unlike unfrozen IgG in acid medium IgG frozen in the same medium did not show increased cathodal electrophoretic migration rate on agarose gel and formed aggregates. IgG frozen in carboxylic acid medium showed, unlike unfrozen IgG in this medium, the same dependence on carbon chain length, as does IgG when frozen at neutral $\mathrm{pH}$ in the presence of amino acids. ${ }^{9}$ The aggregates, formed on freezing of IgG at neutral $\mathrm{pH}$ dissociated in $0.1 \mathrm{M}$ formic acid solutions, but unlike glycine-HCl containing samples, ${ }^{4}$ the formic acid sample aggregated when back dialysed to neutral buffer. The main source of molecules forming these aggregates (Fig. 3) was molecules that migrated towards the cathode. IgG frozen at neutral as well as at acid $\mathrm{pH}$ migrated further in cathodal direction than unfrozen IgG. The results showed an interplay between freezing and acidification in the induction of aggregation of IgG, and showed that groups with partly different charges might be exposed by freezing and by acid treatment.

The findings in this study corroborate the conclusion by Remold and Zimmermann ${ }^{16}$ that the IgG molecules are irreversibly denatured in acid medium. It was thus shown that only $1 / 3$ of the original IgG molecules existed in monomer form after acidification with $1 \mathrm{M}$ formic acid. Solutions containing heavy and light chains from reduced and alkylated IgG are often made $1 \dot{M}$ in respect of carboxylic acids prior to separation. ${ }^{17}$ Isolated heavy chains show the same dependency of their solvent medium ${ }^{6}$ as did the whole IgG molecule. Carboxylic acids acted as irreversible denaturants. The acidification of isolated heavy chains with carboxylic acids would thus result in irreversible conformation changes. These might be larger for heavy chains than for the IgG molecules themselves as the bonds joining the heavy and light chains are important for restricting conformation changes in the presence of acid. ${ }^{13}$ Glycine inhibited the aggregation of both heavy chains ${ }^{6}$ and IgG.4 Glycine could as the light chains be linked to the heavy chains by non-covalent bonds ${ }^{18}$ thereby preserving conformational changes occurring upon acidification. This was in line with the fact that heavy chains obtained from rabbits not immunized with adeno virus inactivate this type of virus when dissolved in glycine- $\mathrm{HCl}$, in spite of the fact that IgG by itself has no inactivating effect. $^{8}$ If this depends on the changes in charge that were seen upon acidification and/or other conformation changes is still too early to say.

At acid $\mathrm{pH}$ but not at neutral $\mathrm{pH}^{9}$ there was a difference in respect of aggregate formation between carboxylic acids and amino acids. Aggregates formed on freezing of IgG at neutral $\mathrm{pH}$ dissociated in carboxylic acid as well as in glycine- $\mathrm{HCl},{ }^{4}$ but carboxylic acid samples aggregated when back dialysed to neutrality. In one respect carboxylic acids were, however, similar to the amino acids. Propionic acid affected native IgG at acid $\mathrm{pH}$ more than did carboxylic acids with carbon-chains of longer or shorter length. The $\mathrm{C}_{3}$ amino acid does not only, as other short chain aliphatic amino acids, inhibit aggregation of IgG by freezing but also dissociates aggregates existing before its addition. ${ }^{9}$

Acknowledgements. This investigation was supported by the Alfred Osterlunds Stiftelse and the Swedish Medical Research Council (Project No. B70-13X-581-06B). 


\section{REFERENCES}

1. Kochwa, S., Smith, E., Brownell, M. and Wasserman, L. R. Biochemistry 5 (1966) 277.

2. Kunkel, H. G., Müller-Eberhard, H. J., Fudenberg, H. H. and Tomasi, I. B. J. Clin. Invest. 40 (1961) 117.

3. Stelos, P. Handbook of Exptl. Immunol., Blackwell, Oxford and Edinburgh 1967, p. 113.

4. Hansson, U.-B. Acta Chem. Scand. 22 (1968) 490.

5. Pressman, D. and Grossberg, A. L. Antibody Specificity, W. A. Benjamin Inc., Now York 1967, p. 211.

6. Kjellén, L. and Hansson, U.-B. Acta Pathol. Microbiol. Scand. 73 (1968) 391.

7. Kjellén, L. and Hansson, U.-B. Unpublished notation.

8. Kjellén, L. and Hansson, U.-B. Virology 40 (1970) 166.

9. Hansson, U.-B. Acta Chem. Scand. 24 (1970) 1585.

10. Laurell, C.-B. Anal. Biochem. 10 (1965) 358.

11. Hansson, U.-B., Laurell, C.-B. and Bachmann, R. Acta Med. Scand. Suppl. 445 (1966) 89.

12. Hansson, U.-B. Acta Chem. Scand. 22 (1968) 483.

13. Ross, D. L. and Jirgensons, B. J. Biol. Chem. 243 (1968) 2829.

14. Stanworth, D. R. and Pardoe, G. I. Handbook of Exptl. Immunol., Blackwell, Oxford and Edinburgh 1967, p. 286.

15. Urnes, P. and Doty, P. Advan. Protein Chem. 16 (1961) 401.

16. Remold, H. and Zimmermann, E. Arch. Biochem. Biophys. 120 (1967) 35.

17. Stanworth, D. R. and Pardoe, G. J. Handbook of Exptl. Immunol., Blackwell, Oxford and Edinburgh 1967, p. 168.

18. Nisonoff, A. and Palmer, J. L. Science 143 (1964) 376.

Received December 24, 1969. 